\title{
Three-Dimensional Gait Analysis of Anterior Cruciate Ligament Before and After Injury
}

Xiaobing Yu, Chunhui Li, Yupeng Liu, Xiaojun Ma and Weiming Wang*

Department of Orthopaedics, Zhongshan Hospital of Dalian University, Dalian, China

*Corresponding author: Weiming Wang, Department of Orthopaedics, Zhongshan Hospital of Dalian University, Dalian, Liaoning province 116001, China, Tel: 86-0411-62893145, 86-0411-13504961988; E-mail: dldxyxb@163.com

Received date: November 30, 2016; Accepted date: January 11, 2017; Published date: January 20, 2017

Copyright: $\odot 2017$ Xiaobing Y, et al. This is an open-access article distributed under the terms of the Creative Commons Attribution License, which permits unrestricted use, distribution, and reproduction in any medium, provided the original author and source are credited.

\begin{abstract}
Objectives: The purpose of this study was to identify clinical reference for the diagnosis of anterior cruciate ligament (ACL) injury. We compared the knee flexion extension rotation angle (FERA), internal and external rotation angle (IERA) and varus eversion rotation angle (VERA) before and after ACL injury.
\end{abstract}

Study design: Cohort study (Diagnosis); Level of evidence II.

Methods: In the control group, 15 health volunteers (male: 6; female: 9) was included. In the ACL injury group, 15 subjects (male: 6; female: 9 ) with ACL injury on single knee were included. Three-dimensional motion system was used to analyze FERA, IERA and VERA during the stance and swing phases of a gait cycle.

Results: The results showed that FERA, IERA and VERA were significantly different between the ACL injury group and the healthy control group. In the swing phase of a gait cycle, knee flexion angle, tibial external rotation and varus reached maximum. In the stance phase of a gait cycle, the extension, tibial internal rotation, varus angles reached maximum. In the healthy control group, FERA, IERA and VERA varied within a narrow range, while in the $A C L$ injury group, FERA, IERA and VERA varied at a significantly larger range.

Conclusions: We concluded that FERA, IERA and VERA can be used as diagnostic reference for ACL injury. Accurate data from gait analysis can provide scientific guidance for postoperative rehabilitation.

Keywords: Anterior cruciate ligament injury; Three dimensional gait; Flexion extension rotation angle; Internal and external rotation angle

\section{Introduction}

Anterior cruciate ligament (ACL) is an important static and stable structure of the knee. Mechanical receptors, present on the surface of $\mathrm{ACL}$, regulate the muscles around the joint structure through the neuromuscular reflection. ACL injuries are common in sports injuries. After ACL injury, alteration in reflex arc can cause compensatory changes in the knee muscles, leading to the changes in gait. This gait change can be measured by professional gait analysis system, and can objectively and quantitatively reflect the functional status of knee during walking [1]. Recently, three-dimensional gait analysis system has been extensively used to analyze the knee movement. With the scientific advancement, three-dimensional gait analysis system is constantly updated and improved [2]. During the gait cycle of normal walking, swing phase and stance phase, the stride, walking frequency, walking length, walking cycle, knee flexion and extension, knee internal and external varus, and tibial internal and external rotation can be exhibited in three-dimensional view. By the comparison of these parameters between ACL injury and normal subjects, the difference of knee from every perspective can be analyzed more intuitively and accurately, which provides basis for the diagnosis, surgical treatment and postoperative rehabilitation exercises of the disease [3].
In this study, we evaluated the characteristic features of gait in ACL injury and healthy groups using three-dimensional gait capturing system.

\section{Materials and Methods}

\section{Clinical data}

In the ACL injury group, there were 15 subjects (male: 6; female: 9) with injuries on the left knee. These subjects were derived from the patients from January 2015 to December 2015 and the injury time ranged from 3 to 24 months (Table 1). After the injury, all subjects did not undergo surgery.

\begin{tabular}{|l|l|l|l|l|}
\hline Gender & N & Age (years & $\begin{array}{l}\text { Body weight } \\
\mathbf{( K g}\end{array}$ & Body length(cm) \\
\hline Male & 6 & $26.4 \pm 3.7$ & $58 \pm 5.6$ & $169.1 \pm 8.9$ \\
\hline Female & 9 & $25.1 \pm 3.3$ & $48 \pm 5.8$ & $158.2 \pm 6$ \\
\hline
\end{tabular}

Table 1: Basic information of the normal subjects.

Arthroscopic examination showed that ACL was completely broken in all subjects. There were no previous other knee diseases, no collateral ligament complete rupture, and no posterior cruciate ligament injury. Anterior drawer test, Lachman test, and pivot shift test 
Page 2 of 5

were positive. Contralateral limbs were negative as examined by both general physical test and sports medical test. All subjects had no history of trauma and joint diseases. During walking, there was no knee pain and interlocking symptoms.

In the control group, a total of 15 healthy volunteers derived from our Clinical School Students (Table 2). The subjects were informed with the study method and purpose before the test and informed consent was signed. All subjects had no previous other knee disorders. General physical examination and sports physical examination were negative. There was no history of hip, knee, ankle disease or a history of trauma, central nervous system disease and no history of back pain. The subjects were not familiar with treadmill walking, and there were no pathological conditions affecting the lower limb gait.

\begin{tabular}{|l|l|l|l|l|}
\hline Gender & N & Ageyears & Body Weight $\mathbf{( k g}$ & Body length(cm) \\
\hline Male & 6 & $27.2 \pm 4.7$ & $58 \pm 5.6$ & $169.1 \pm 8.9$ \\
\hline Female & 9 & $26.1 \pm 6.3$ & $50 \pm 4.3$ & $158.2 \pm 6.8$ \\
\hline
\end{tabular}

Table 2: Basic information of the subjects with ACL injury, the age, body weight and body length are similar between the two groups.

\section{Test methods}

The passive optical MoCap system (DVMC ${ }^{\oplus}$-8820) had a sampling frequency of $60 \mathrm{~Hz}$ with an absolute error $<1 \mathrm{~mm}$. The system consists of three parts: (1) 12 infrared cameras distributed at different positions; (2) optical markers attached to the test site; and (3) computer and other equipment that regulate synchronous operation of the other two parts and analyze the data. First, the gait was adjusted and the configuration between the main equipment and infrared cameras as well as the measuring range were analyzed. The lower limbs were tied with two elastic bands. The markers were glued to the bands and form an equilateral triangle in order to ensure that the markers indicate the position of the limb during movement. The reflection points were not blocked by the clothes. The following reflex points were used for three-dimensional gait analysis: (1) waist and abdomen pelvis: anterior superior iliac spine, sacrum (3 points); (2) hip: greater trochanter (2 points); (3) knee: the lateral femoral condyle, tibial tuberosity and fibular head (8 points); (4) the ankle: internal and external ankle tips (4 points); and (5) the areas between the first and second tarsometatarsal (2 points). The treadmill (ECON USA) walking area was in the infrared camera capturing area. The treadmill belt had an area of $480 \mathrm{~mm} \times 1400 \mathrm{~mm}$. The subjects walked for $6 \mathrm{~min}$ on the treadmill at conditions of natural and customary posture and at the speed $1.8 \mathrm{~km} / \mathrm{h}$.

\section{Parameters}

The kinematic and kinetic parameters include: step length, step width, FERA, IERA, and VERA. The parameters were measured for 10 consecutive gait cycles with stable posture during treadmill walking. Average and standard deviation were calculated for each parameter.

\section{Statistical analysis}

In this study, in order to ensure the accuracy and authenticity of the gait data, MotAnaly software was applied to eliminate the head and tail data for each group. The processed data were from the beginning to the end of each gait cycle. Two-sample t-test was used to compare each parameter between the control and ACL injury groups using SPSS18.0 statistical software. $\mathrm{P}<0.05$ was considered significant difference.

\section{Results}

\section{Knee angular movement curve}

During walking, FERA was $-2^{\circ} \sim 66^{\circ}$, IERA was $9^{\circ} \sim-5^{\circ}$ and VERA was $8^{\circ} \sim-4^{\circ}$ for healthy subjects. In the stance phase, when the heel was off the ground, the maximum knee extension angle, maximum knee varus and the maximum external rotation occurred. In this phase, flexion angle did not change significantly. In mid-accelerating swing phase, maximum knee flexion angle was achieved. Almost at the same time, the maximum internal rotation and maximum eversion occurred. In the ACL injury group, FERA was $-5^{\circ} \sim 52^{\circ}$, IERA was $24^{\circ} \sim$ $-3^{\circ}$ and VERA was $14^{\circ} \sim-13^{\circ}$. With the increase in the angle of knee flexion and extension, the external rotation angle and varus angle also increased. In the stance phase, when the heel was off the ground, the knee extension angle reached maximum. At the same time, the maximum eversion and maximum internal rotation occurred. In the swing phase with accelerating speed, knee flexion angle reached maximum, followed by the occurrence of the largest knee varus and external rotation (Figure 1).

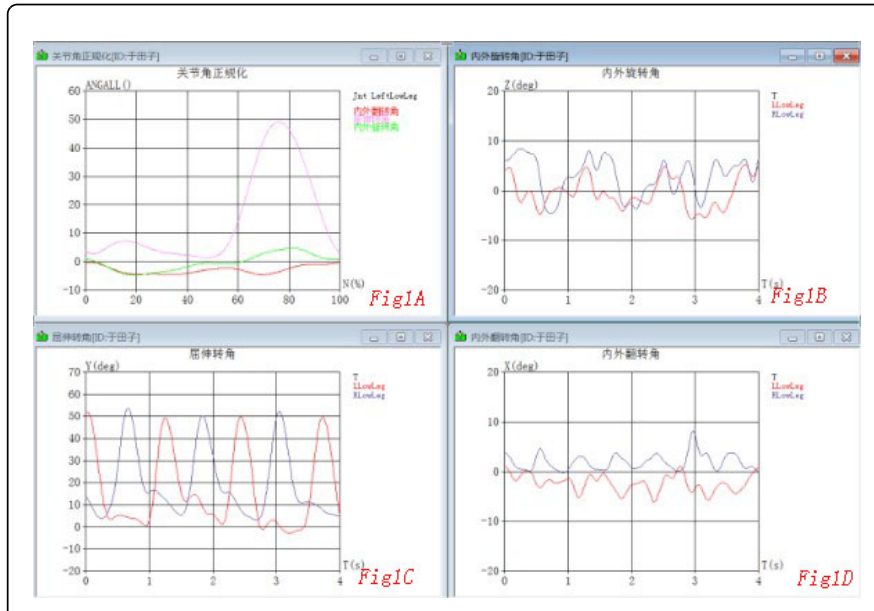

Figure 1: Variation in the varus and rotation angle during normal walking. Figure 1A: Joint angle curve after normalization. Figures 1B, 1C, 1D: IERA, FERA, VERA respectively.

\section{Knee angle parameters in the three-dimensional gait}

During the gait cycle, both control and ACL injury groups exhibited the cycle of knee flexion, knee extension and knee flexion. In the swing phase, the maximum knee flexion angle for ACL injury and control groups was $40.0 \pm 8.2^{\circ}$ and $60.4 \pm 5.9^{\circ}$, respectively. The maximum knee flexion angle in ACL injury group was significantly smaller than that in the control group (P0.05). In the swing phase, the maximum varus angle in the ACL injury group was $3.9 \pm 1.1^{\circ}$, which was significantly larger than that in the control group $\left(2.8 \pm 6.9^{\circ}\right)$ (Table 3 ). The maximum external rotation angle in ACL injury group was $12.9 \pm$ $1.3^{\circ}$, which was significantly larger than that in the control group (2.9 $\left.\pm 2.0^{\circ}\right)($ P0.05) (Figure 2 and Table 3$)$. 
Citation: Xiaobing Yu, Chunhui Li, Yupeng Liu, Xiaojun Ma, Weiming Wang (2017) Three-Dimensional Gait Analysis of Anterior Cruciate Ligament Before and After Injury. J Bioengineer \& Biomedical Sci 7: 215. doi:10.4172/2155-9538.1000215

Page 3 of 5

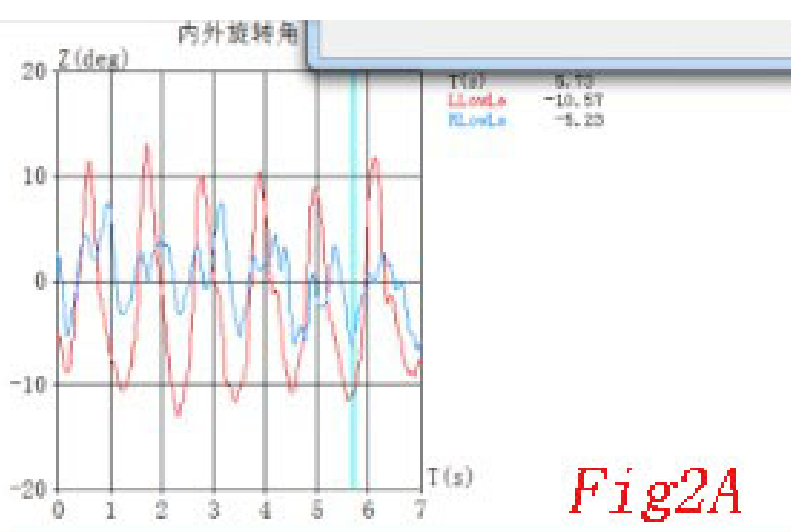

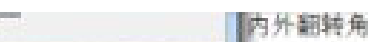

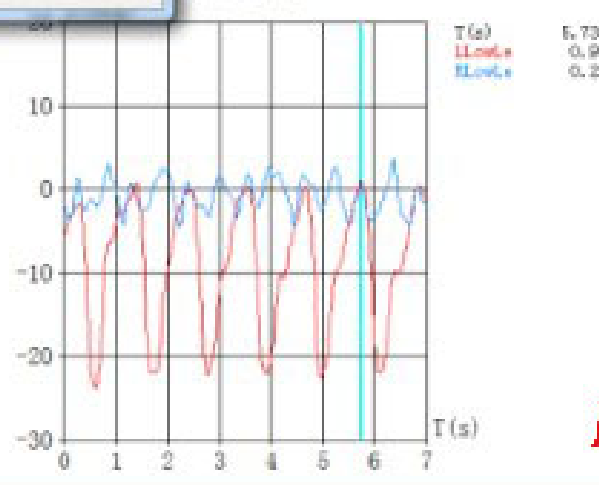

Fig2B
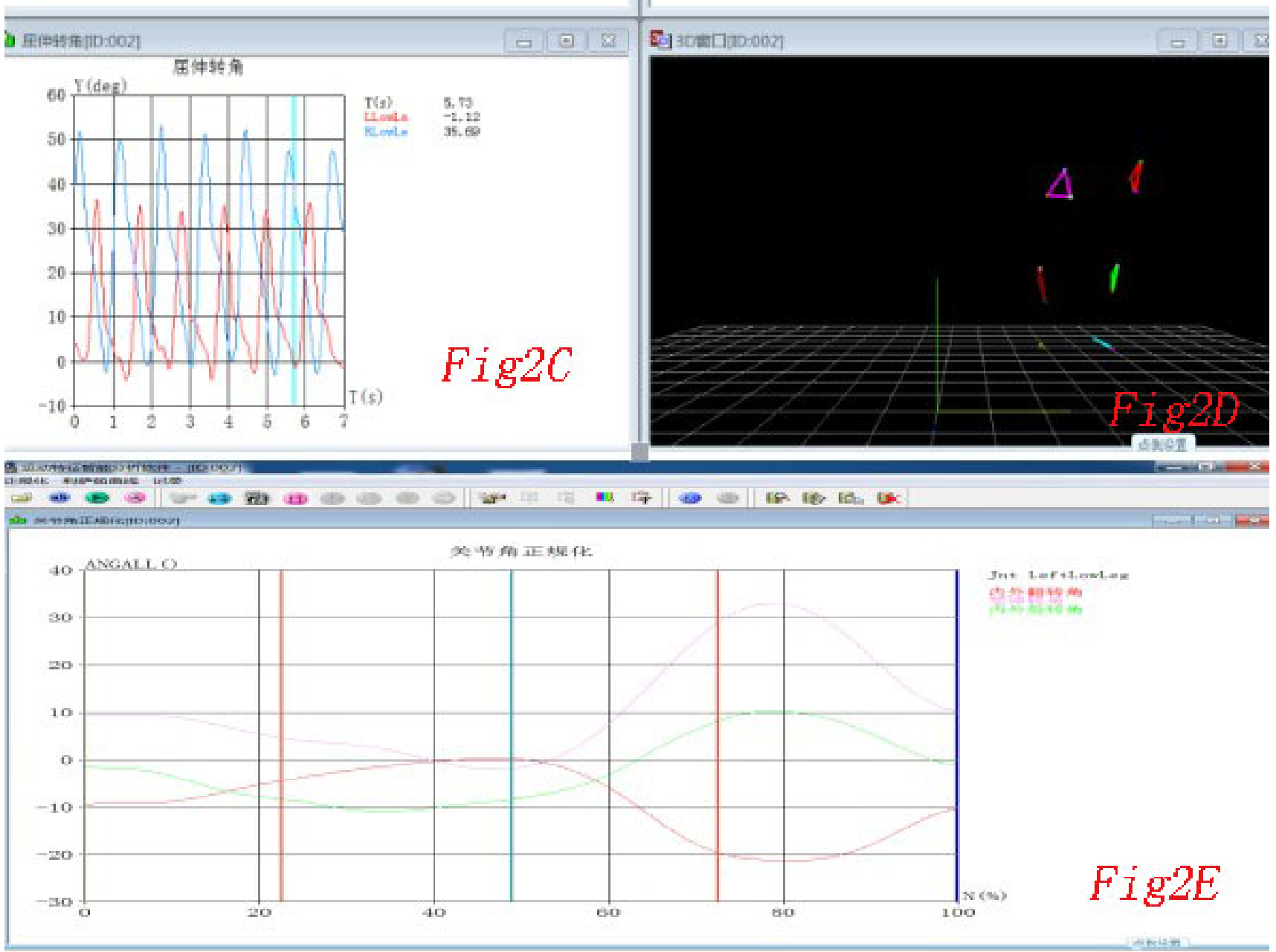

Figure 2: Variation of varus and tibial rotation angle during knee extension and flexion in the subjects with ACL injury. Figure 2A, 2B, 2C: IERA, VERA, FERA respectively. Figure 2D: The gait of a $3 \mathrm{~d}$ window display. Figure 2E: Joint angle curve after normalization.

\begin{tabular}{|l|l|l|l|l|}
\hline Angle parameters & ACL injury group & Normal control group & $\mathbf{t}$ & $\mathbf{P}$ \\
\hline Flexion angle & $40.5 \pm 5.15 .5$ & $60.4 \pm 5.95 .5$ & 5.46 & 0.01 \\
\hline Extension angle & $6.7 \pm 1.70 .6$ & $1.8 \pm 1.10 .9$ & 2.11 & 0.01 \\
\hline
\end{tabular}




\begin{tabular}{|l|l|l|l|l|}
\hline Varus angle & $3.9 \pm 1.17 .8$ & $2.8 \pm 6.98 .9$ & 1.01 & 0.01 \\
\hline Eversion angle & $19.10 \pm 3.06(5.4$ & $5.08 \pm 4.054 .6$ & 1.47 & 0.01 \\
\hline Internal rotation angle & $13.1 \pm 1.8(2.8$ & $6.1 \pm 2.03 .2$ & 1.32 & 0.01 \\
\hline External rotation angle & $12.9 \pm 1.34 .8$ & $2.9 \pm 2.00 .9$ & 1.29 & 0.01 \\
\hline
\end{tabular}

Table 3: Parameters for the angles in the normal and ACL injury groups $\left(\mathrm{X}^{\wedge}- \pm \mathrm{s}(\mathrm{CV})\right)$.

\section{Discussion and Conclusion}

In previous studies, hip, knee, and ankle were analyzed together [4]. In this study, we individually analyzed knee parameters during weightbearing walking on the ground. We collected three-dimensional gait motion data for both healthy and patients with ACL injury. Previous three-dimensional motion studies usually focused on a single sagittal plane motion, while each angle of the knee cannot be analyzed together. Other studies performed physical examinations, e.g., X-ray, B ultrasound, MRI when the subjects are under passive conditions without weight-bearing. All these previous reports do not carry out studies under the gait condition and do not collect data during walking. In this study, we analyzed the knee flexion and extension, varus, internal and external rotation under active, weight-bearing condition. Furthermore, we directly presented the data as a curve by computer, which is different from previous studies that usually only presented data pattern [5].

Most gait and motion analysis equipment using light and electricity to capture skin marker has limitations because the markers are not easily affixed to the skin and they are easily lost during the threedimensional capture. Repeated gait acquisition causes unnecessary trouble to the patients [6-8]. We made markers on elastic bandage that are attached closely to the skin. Furthermore, the triangle markers are relatively stable, which controls the error within a certain range, and produces the highest accuracy. The main limitation for this method is that the soft tissue wrapped with bandages produces relative displacement with the bone position. It has been reported that it is more accurate by equipping the markers on a Kirschner that is placed into the femur and tibia. However, this method causes pain and generally people do not accept invasive test. Furthermore, this method cannot be used in patients' preoperative evaluation [9-11]. In this study, we analyzed the three-dimensional gait, calculated FERA, IERA and VERA in each gait cycle and obtained the curve for these three angles for both healthy subjects and patients with ACL injury. We found that these three angles were significantly different between the healthy and ACL injury groups. Increases in the knee rotation angle and varus angle resulted in the instability of ACL. The results for the angles obtained in this study were consistent with previous reports [12]. We confirmed that during walking, the motion of knee is not simply on the sagittal plane. Instead, the knee sagittal plane motion is accompanied by varus and rotation, which is namely three planes of motion. Furthermore, there is certain pattern of the three planes of motions for both healthy subjects and patients with ACL injury. Some studies showed that after ACL injury, the knee angle produces adaptive compensatory changes under the neuromuscular regulation. In the future studies, we will combine with neuromuscular research to provide comprehensive analysis of the pattern of three-dimensional knee motion. For each subject, maximum angles of flexion, varus, and rotation were different, but the phase to reach the maximum angle is the same. The variation among the three angles is also consistent. In the stance phase of healthy subjects, when the heel is on the ground, knee is eversion, and then the eversion angle was reduced and transitioned to varus. The knee internal rotation angle decreased and transitioned into external rotation. Maximum knee extension angle was achieved right before the toe was off the ground. In the swing phase, the knee flexion reached the maximum after the toe was off the ground. With increases in knee flexion angle, knee varus transitioned to eversion and the limb external rotation transitioned to internal rotation. In the ACL injury group, the knee is varus when the heel is on the ground. Subsequently, the varus angle is reduced and transitioned to eversion. The knee external rotation angle is reduced and transitioned to internal rotation angle. The knee is changed from flexion to extension. The extension angle reached maximum before the toe is off the ground. In the swing phase, the extension is transitioned to flexion after the toe is off the ground, and the flexion angle reached maximum in the entire gait cycle. With the increases in the flexion angle, knee is transitioned from eversion to varus and the limb is transitioned from internal rotation to external rotation.

In this study, the extension angle during the stance phase in the ACL injury group was increased compared to that in the control group, while the flexion angle during the swing phase was decreased compared to that in the control group. The decreases of the flexion angle may be due to the tibia forward moving caused by quadriceps activity after injury, knee instability, pain and discomfort. The increases in the varus angle and the rotation angle in the ACL injury group may be due to the disappearance of ACL-controlled rotation and the antitibia stretching activity of quadriceps. It may be associated with the action of quadriceps, and posterior cruciate ligament. The increases in the varus angle and the rotation angle also indicate knee instability.

\section{Conflict of Interest}

Authors have no conflicts of interest.

\section{Acknowledgments}

We thank all patients and theirs family.

\section{References}

1. Webster KE, Wittwer JE, O’Brien J, Julian AF (2005) Gait patterns after anterior cruciate ligament reconstruction are related to graft type. Am J Sports Med 33: 247-254.

2. Knoll Z, Kocsis L, Kiss RM (2004) Gait patterns before and after anterior cruciate ligament reconstruction. Knee Surg Sports Traumatol Arthrosc 12: 7-14.

3. Andriacchi TP (1993) Functional analysis of pre and post knee surgery; total knee arthrosplasty and ACL reconstruction. J Biomech Eng 115: $575-581$. 
Citation: Xiaobing Yu, Chunhui Li, Yupeng Liu, Xiaojun Ma, Weiming Wang (2017) Three-Dimensional Gait Analysis of Anterior Cruciate Ligament Before and After Injury. J Bioengineer \& Biomedical Sci 7: 215. doi:10.4172/2155-9538.1000215

Page 5 of 5

4. Hurwitz DE, Andriacchi TP, Bush-Joseph CA, Bach Bernard R (1997) Functional adaptations in patients with ACL-deficient knee. Exerc Sport Sci Rev 25: 1-20.

5. Berchuck M, Andriacchi TP, Bach BR, Reider B (1990) Gait adaptations by patients who have a deficient anterior cruciate ligament (ACL). J Bone Joint Surg Am 72: 871-877.

6. Wexler G, Hurwitz DE, Bush-Joseph CA, Andriacchi TP, Bach Bernard R (1998) Functional gait adaptations in patients with anterior cruciate ligament deficiency over time. Clin Orthop 348: 166-175.

7. Knoll Z, Kiss RM, Kocsis L (2004) Gait adaptation in ACL deficient patients before and after anterior cruciate ligament reconstruction surgery. J Electromyogr Kinesiol 14: 287 -294.

8. Roberts CS, Rash GS, Honaker JT, Wachowiak PM, John CS (1999) A deficient anterior cruciate ligament does not lead to quadriceps avoidance gait. Gait Posture 10: 189-199.
9. Rudolph KS, Eastlack ME, Axe MJ (1998) Movement patterns after anterior cruciate ligament injury: A comparison of patients who compensate well for the injury and those who require operative stabilization. J Electromyogr Kinesiol 8: 349-362.

10. Devita P, Hortobagyi T, Barrier J (1998) Gait biomechanics are not normal after anterior cruciate ligament reconstruction and accelerated rehabilitation. Med Sci Sports Exerc 30: 1481-1488.

11. Bush-Joseph CA, Hurwitz DE, Patel RR, Yersin B, Ralph Garretson, et al. (2001) Dynamic function after anterior cruciate ligament reconstruction with autologous patellar tendon. Am J Sports Med 29: 36-41.

12. Timoney JM, Inman WS, Quesada PM, Peter FS, Robert LB (1993) Return of normal gait patterns after anterior cruciate ligament reconstruction. Am J Sports Med 21: 887-889. 